\title{
Adenovirus is prevalent in juvenile polyps and correlates with low vitamin D receptor expression
}

\author{
Lingling Wang ${ }^{1,2,6}$, Hongmei Guo ${ }^{3,6}$, Jingwen $\mathrm{Li}^{4}$, Susu $\mathrm{He}^{1,2}$, Guang Yang ${ }^{3 凶}$ and Erguang $\mathrm{Li}^{1,2,5^{凶}}$ \\ (c) The Author(s), under exclusive licence to the International Pediatric Research Foundation, Inc 2021
}

BACKGROUND: The objective of this study was to assess human adenovirus (HAdV) infection in juvenile polyps (JPs) and to preliminarily establish a correlation to vitamin D receptor (VDR) expression.

METHODS: The study includes 76 patients of $5.2 \pm 2.8$ years old. Seventy-eight JP specimens and 24 parapolyp tissues from polypectomy were used. PCR was used to detect HAdV DNA and quantitative reverse transcription-PCR for viral and host gene expression. The PCR products were sequenced for virus typing. The correlation between VDR expression and HAdV infection was established using nonparametric Spearman's analysis.

RESULTS: Seventy-four children (97.4\%) had a single polyp and two had two polyps. The histopathological characteristics of the polyps were in line with JP. Thirty-three samples had HAdV DNA (43.4\%), including 32 subgroup C and 1 subgroup B HAdV; no enteric HAdV was detected. HAdV messenger RNA was detected in 5 of the 33 samples (15.2\%). The samples had increased interleukin-1 $\beta$ (IL-1 $\beta)$, IL-6, and calprotectin expression, and reduced E-cadherin and VDR expression. JP samples with low VDR expression were more prevalent of HAdV DNA ( $r=1.261,95 \%$ confidence interval, 1.017-1.563), while VDR expression positively correlated with E-cadherin and negatively with inflammation gene expression.

CONCLUSIONS: HAdV latent infection was prevalent among JP tissues. The presence of HAdV correlated positively to low VDR expression.

Pediatric Research (2022) 91:1703-1708; https://doi.org/10.1038/s41390-021-01697-y

\section{IMPACT:}

- The HAdVs infect the upper airways and gastrointestinal system and is found to persist in lymphoid tissues. The prevalence of HAdV and the status of the infection is unknown.

- The study investigated the prevalence of HAdV from polypectomy specimens of JP patients and found that HAdV was prevalent and was in a persistent state. HAdV infection was more prevalent in samples with low VDR expression.

- Whether HAdV infection and reactivation is a contributing factor to JPs is unknown. Factors such as proinflammation and bacterial metabolites that are known to promote HAdV reactivation warrant further investigation.

\section{INTRODUCTION}

Juvenile polyps (JPs) are benign intestinal polyps formed by rounded mucosal hamartoma of the lamina propria in the large bowel. Also known as congenital or retention polyps, these types of polyps develop in the rectum or sigmoid colon of children $<5$ years of age. ${ }^{1-3}$ The polyps are solitary in most cases, but can be a few in some patients. ${ }^{4}$ JPs often remain asymptomatic with gastrointestinal (Gl) bleeding, an indicative symptom. Thus, the most common presenting symptom of JPs is bleeding that results from ulceration of the polyp surface. Blood loss is usually minimal and appears as bright red streaks of blood over the surface of the stool. ${ }^{5}$ Another character of JPs is that they will autoamputate, resulting in spontaneous cessation of rectal bleeding. ${ }^{2}$ Although the incidence of JPs is unknown, they are believed to occur in $\sim 1-2 \%$ of all preschool- aged children, with the peak incidence between 3 and 5 years of age. With increases in the demand for colonoscopy, the detection rate of JPs can be high. Some studies have reported that polyps were found in $7-27.2 \%$ of children undergoing colonoscopy.,

The etiology of JPs is unknown. Studies link JPs to factors such as hereditary, genetic, and inflammatory causes. ${ }^{2}$ The connection between virus infection and JPs is rarely reported, although both viral and bacterial infection is among the most common causes of gastroenteric diseases in children. Microscopically, the main body of the polyp consists of dilated or cystic epithelial tubules that are lined by normal colonic epithelium. ${ }^{7}$ It is generally considered that polyps are the result of ulceration and subsequent inflammation of the mucosa, causing obstruction of regional, small colonic glands of the mucosa. The obstructed glands proliferate, branch,

\footnotetext{
${ }^{1}$ SKL of Pharmaceutical Biotechnology, Medical School of Nanjing University, Nanjing, China. ${ }^{2}$ Jiangsu Key Laboratory of Molecular Medicine, Medical School of Nanjing University, Nanjing, China. ${ }^{3}$ Nanjing Children's Hospital, Nanjing Medical University, Nanjing, China. ${ }^{4}$ Changzhou \#2 People's Hospital, Nanjing Medical University, Nanjing, China. ${ }^{5}$ Shenzhen Research Institute of Nanjing University, Shenzhen, China. ${ }^{6}$ These authors contributed equally: Lingling Wang, Hongmei Guo. ${ }^{凶}$ email: gyangnj@qq.com; erguang@nju.edu.cn
}

Received: 25 January 2021 Revised: 19 May 2021 Accepted: 9 July 2021

Published online: 16 August 2021 
Table 1. Patient information and test results.

\begin{tabular}{|c|c|}
\hline Characteristics & Information \\
\hline Patients $(n)$ & 76 \\
\hline Gender (male/female) & $57 / 19$ \\
\hline Age $($ mean \pm SEM) & $5.6 \pm 2.8$ \\
\hline$<3$ years $(n)$ & 18 \\
\hline $3-<5$ & 33 \\
\hline $5-<8$ & 17 \\
\hline$\geq 8$ & 8 \\
\hline \multicolumn{2}{|l|}{ Polyp location (n) } \\
\hline Rectum & 50 \\
\hline Sigmoid colon & 23 \\
\hline Other areas & 5 \\
\hline \multicolumn{2}{|l|}{ JP size $(n)$} \\
\hline$<1 \mathrm{~cm}$ & 32 \\
\hline $1-2 \mathrm{~cm}$ & 24 \\
\hline$\geq 2 \mathrm{~cm}$ & 22 \\
\hline Ad status (positive, patients, $\%)^{a}$ & $33,76,43.4 \%$ \\
\hline$<3$ years & $7,18,38.9 \%$ \\
\hline $3-<5$ years & $15,33,45.4 \%$ \\
\hline $5-<8$ & $7,17,41.2 \%$ \\
\hline$\geq 8$ & $4,8,50.0 \%$ \\
\hline Rectum & $17,48,35.4 \%$ \\
\hline Sigmoid colon & $13,23,56.5 \%$ \\
\hline Other areas & $3,5,60 \%$ \\
\hline \multicolumn{2}{|l|}{ Adenovirus species or serotype ${ }^{b}$} \\
\hline Species C & 32 \\
\hline Species B & 1 \\
\hline HAdV40 or 41 & 0 \\
\hline $25(\mathrm{OH}) \mathrm{D}(\mathrm{nM}, \text { mean } \pm \mathrm{SEM})^{\mathrm{c}}$ & $59.6 \pm 9.7$ \\
\hline Normal (>75 nM) & $2,7.4 \%$ \\
\hline Insufficient (50-75 nM) & $19,70.4 \%$ \\
\hline Deficient $(<50 \mathrm{nM})$ & $6,22.2 \%$ \\
\hline
\end{tabular}

${ }^{a}$ We collected 78 polyps from 76 patients. Two patients had two polyps removed, but only one polyp was tested for HAdV.

${ }^{\mathrm{b}}$ The variable region of the hexon gene was used for species classification. 'Blood 25-hydroxyvitamin D (25(OH)D) levels among patients $(n=27)$ are listed.

and dilate, forming a cystic structure that will eventually push up the mucosa, leading to further inflammation, ulceration, and formation of granulation tissue. The inflammation and ulceration increase the exposure to bacteria and viruses. The human adenovirus (HAdV) is among the common pathogens that infect the upper airways and the Gl system and is found to persist in lymphoid tissues. The persistence of HAdV in the Gl tract was identified in $31 \%$ of children, ${ }^{8,9}$ while virus infections, particularly subgroup $C$ adenoviruses, are commonly detected among patients with intestinal intussusception. ${ }^{10-13}$ The HAdVs are so named based on their isolation from resected adenoids. ${ }^{14-17}$ They are known for a tendency to acutely infect the upper respiratory tract and the Gl tract and to persist in lymphoid tissues. ${ }^{18,19}$ The persistence or reactivation of HAdV in the Gl tract is likely a common event since persistent adenoviral shedding in stools is known to occur past convalescence following acute adenoviral infections. $^{20-23}$ In some cases, asymptomatic HAdV shedding in the stools can last for over 2 years after the primary infection. ${ }^{24}$
The prevalence and status of HAdV in JPs have not been investigated.

We studied JP specimens from 76 patients and found that HAdV DNA was prevalent among the samples. In addition, the virus seemed to remain in nonreplicative state since we only detected HAdV messenger RNA (mRNA) from $\sim 15.2 \%$ of the samples. Here we report the investigation of HAdV infection in JP samples.

\section{MATERIALS AND METHODS}

\section{Tissues samples and stool samples}

Tissue and stool samples were collected as part of the routine operation for patients undergoing colonoscopy or sigmoidoscopy for the diagnosis and treatment of JPs. The studies were approved by the Ethics Committees of Nanjing Medical University and Nanjing University Medical School, and informed consent was obtained from parents or legal guardians in accordance with the Declaration of Helsinki. The study involved 76 patients (57 males and 19 females) with 78 JPs, 24 parapolyp specimens, and stool samples collected from June 2016 to June 2018 at the Department of Gastroenterology, Nanjing Children's Hospital. The information of the patients is summarized in Table 1.

\section{DNA extraction and HAdV detection}

JP tissue samples $(\sim 20 \mathrm{mg})$ were homogenized in $100 \mu \mathrm{l}$ buffer containing 150 $\mathrm{mM} \mathrm{NaCl}, 0.5 \%$ NP-40, $0.1 \%$ SDS, and $20 \mathrm{mM}$ Tris- $\mathrm{HCl}$ (pH 7.6). The samples were centrifuged at $13,000 \times g$ to remove cellular debris. Stool samples $(50 \mathrm{mg})$ were diluted 1:10 with lysis buffer and centrifuged at $13,000 \times g$ for $3 \mathrm{~min}$ to collect the supernatants. For DNA extraction, the supernatants of JP tissue lysates or stool samples were treated with proteinase $\mathrm{K}(0.2 \mathrm{mg} / \mathrm{ml})$ at $55^{\circ} \mathrm{C}$ overnight to deproteinate viral DNA associated proteins. The DNA was then purified by phenol-chloroform extraction, followed by ethanol precipitation. Total DNA was resuspended in a Tris-EDTA (TE) buffer and the concentrations were determined with a Nanodrop 2000 instrument. DNA was used as a template for HAdV detection by nested PCR as previously reported. ${ }^{16,25}$ Briefly, the first PCR reaction was performed using a pair of degenerate oligos (hex1deg, 5'-GCCSCARTGGKCWTACATGCACATC-3' and hex2deg, 5'-CAGCACSCCICGRATGTCAAA-3') in a final volume of $50 \mu \mathrm{l}$ containing $100 \mathrm{ng}$ of DNA template. The reaction conditions were as follows: $94^{\circ} \mathrm{C}$ for $3 \mathrm{~min}$, followed by 35 cycles of $94^{\circ} \mathrm{C}$ for $30 \mathrm{~s}, 55^{\circ} \mathrm{C}$ for $30 \mathrm{~s}$, and $72^{\circ} \mathrm{C}$ for $1 \mathrm{~min}$, and a final extension at $72^{\circ} \mathrm{C}$ for $5 \mathrm{~min}$. For the second PCR (nested reaction), onetenth of the PCR mixture was amplified using the same parameters described above, with the following primers, respectively, nehex3deg, $5^{\prime}$-GCCCGYGCMACIGAIACSTACTTC- $3^{\prime}$ and nehex4deg, 5'-CCYACRGCCAGIGTRWAICGMRCYTTGTA- $3^{\prime}$. The amplified products were electrophoresed and were sequenced by an automated DNA analyzer (Sangong Biotech, Nanjing, China). The sequences were subjected to BLAST searching against the GenBank database.

\section{RNA extraction and quantitative reverse transcription-PCR} (RT-qPCR)

Total RNAs were extracted with TRIzol reagent (Ambion). RT was performed with $1 \mu \mathrm{g}$ of total RNA using HiScript III RT SuperMix (Vazyme). The complementary DNA was examined by PCR using primers that anneal to the hexon gene, a conserved region of the species $C$ or $B$ adenovirus as previously reported for HAdV mRNA or for VDR, IL-1 $\beta$, IL-6, and E-cadherin expression by RT-qPCR with 7300 Real-Time PCR System (Thermo Fisher). Cellular glyceraldehyde 3-phosphate dehydrogenase (GAPDH) was used for normalization. The data were analyzed using the $2^{-\Delta \Delta C t}$ method for relative quantification following the MIQE guidelines. ${ }^{26}$ The primer sequences are listed in Table 2.

\section{Measurement of 25-hydroxyvitamin D (25(OH)D) by enzyme- linked immunoassay}

Blood 25(OH)D was determined in the hospital using an EIA Kit (IDS, Boldon, UK) by following the manufacturer's instructions. Serum 25(OH)D reflects both vitamin $\mathrm{D}$ metabolic status and the contributions from all sources of vitamin D. ${ }^{27}$ Age- and sex-matched healthy infants whose blood samples were collected around the same time frame at the Child Health Clinic of the Hospital were included as controls. 
Table 2. Oligo sequences used for detection of gene expression ${ }^{\mathrm{a}}$.

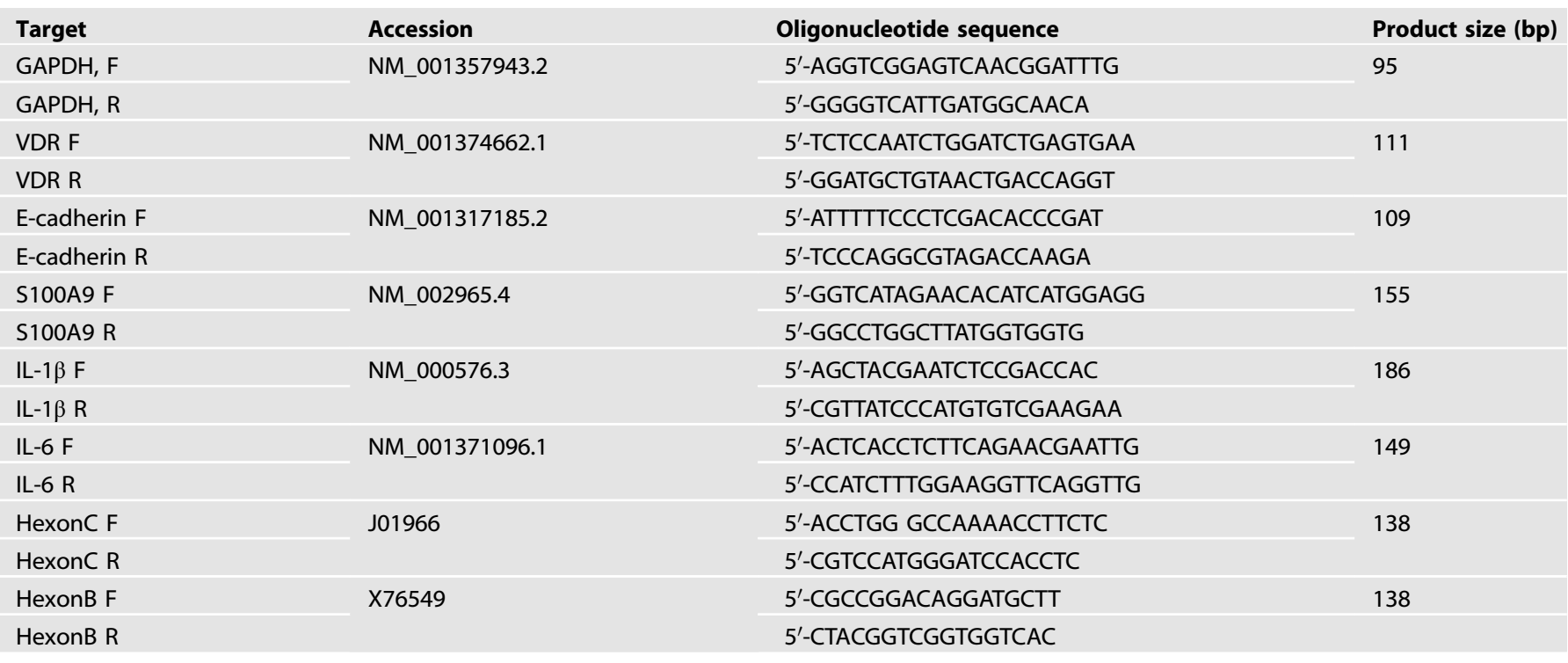

${ }^{\mathrm{a}}$ Gene accession number of corresponding genes is given. The S100A9 subunit was used for the detection of calprotectin.

\section{Statistical analysis}

Statistical analysis was done with SPSS. An independent two-sample $t$ test was used to analyze parametric continuous variables, and a crosstabulation analysis with the $X^{2}$ test was used to analyze nominal variables. Because of our relatively small sample sizes, we used a nonparametric Spearman's correlation analysis to determine the correlation between the real level of VDR, IL-1 $\beta, I L-6$, calprotectin, and E-cadherin expression and the HAdV-positive JPs. A Pearson's correlation test was utilized to address the correlation between VDR and that of IL-1 $\beta, \mathrm{IL}-6$, calprotectin, and E-cadherin expression. A $P$ value $\leq 0.05$ was considered statistically significant.

\section{RESULTS \\ Study participants}

The samples, including 78 polyp samples and 24 parapolyp specimens, were collected from 76 patients undergoing colonoscopy or sigmoidoscopy for the diagnosis and treatment of JPs during June 2016 and June 2018 at the Department of Gastroenterology, Nanjing Children's Hospital. A protocol for the use of the samples was approved by the Clinical Research Ethics Committee of Nanjing Children's Hospital. The intestinal polyp samples were from children aged from 16 months to 14.5 years old, with an average of $5.2 \pm 2.8$ years of age. Among the 76 children, 57 were male and 19 were female. Most of the children had blood in their stools $(92.1 \%), 6$ patients had prolapse of anal mass. Seventy-four children (97.4\%) had a single polyp and two had two polyps. The polyps were located in the rectum (63.2\%), sigmoid colon (30.3\%), and transverse colon or descending colon (6.6\%). The 78 polyps varied in sizes from $<1 \mathrm{~cm}$ (32 cases, $41.0 \%$ ) to $1-2 \mathrm{~cm}$ (24 cases, $30.8 \%$ ), and $>2 \mathrm{~cm}$ ( 22 cases, $28.2 \%$ ). The histopathology of the polyps was in line with the histopathological characteristics of JPs, including cystic architecture, mucus-filled glands, and prominent lamina propria, through resection under the microscope.

A retrospective review of the JP patients with $(25(\mathrm{OH}) \mathrm{D})$ status tested indicated that the patients generally had lower levels of serum 25(OH)D than healthy individuals. 25(OH)D levels in the patients were at $59.6 \pm 9.7 \mathrm{nM}$, compared to that of $77.8 \pm 7.6 \mathrm{nM}$ from age-paired controls of children who attended the outpatient clinic for health check-ups.

\section{Detection and identification of adenoviral DNA}

We performed a PCR study to determine whether the samples had HAdV DNA using a pair of universal primers that target the conserved region of the hexon gene of HAdVs. ${ }^{25}$ The amplicons were then subjected to automated sequence analysis. HAdV DNA was detected in $43.4 \%$ of the patients (33 in 76 patients). The majority of the DNA belonged to subgroup $C$ virus (32 of 33 samples) and one to subgroup B. No HAd40 or HAd41 DNA was detected from the samples. In addition, the prevalence of HAdV among different age groups seemed to be evenly distributed.

Adenovirus can exist in a lytic cycle or in latent status. We performed RT-PCR studies to determine Ad gene transcription by RT-PCR. Although adenoviral DNA was detected from $43.4 \%$ of patients, only five of the samples had adenoviral RNA detected (15.2\%), indicating that the majority of HAdV remained nonreplicative state in the JP tissues. This is consistent with the result from previous studies of stool samples. ${ }^{13,23,24}$ In a follow-up study, we surveyed the stool samples of the patients and found a relatively low incidence rate in virus shedding from the patients. We detected adenoviral DNA from $~ 4 \%$ of the stool samples of patients who were going to have endoscopy examinations. This result further supports the notion that most of HAdVs were latent in the JP tissues.

We detected species $\mathrm{CHAdVs}$ as a predominant subgroup in JPs. While HAdV40 and HAdV41 are reported as enteric pathogens, nonenteric HAdVs, especially subgroup $\mathrm{C}$ viruses, are commonly detected from gastric specimens. ${ }^{10,11,28,29}$ In addition, the results suggested that HAdVs in the JP tissues were in a nonreplicative stage in most of the cases. Many factors such as bacterial products like short-chain fatty acids may reactivate HAdV from latency. ${ }^{30,31}$ Whether virus reactivation modifies JP disease remains uninvestigated.

\section{Correlation of HAdV detection to VDR expression}

Accumulating evidence suggests that vitamin D insufficiency could potentially attribute to viral and bacterial infections. We noticed that the patients, in general, had insufficient or deficient $25(\mathrm{OH}) \mathrm{D}$ levels in their blood. We, therefore, performed RT-qPCR study to determine VDR gene expression in the JP and the parapolyp tissues since the active form of vitamin D exerts the function through VDR. We found 

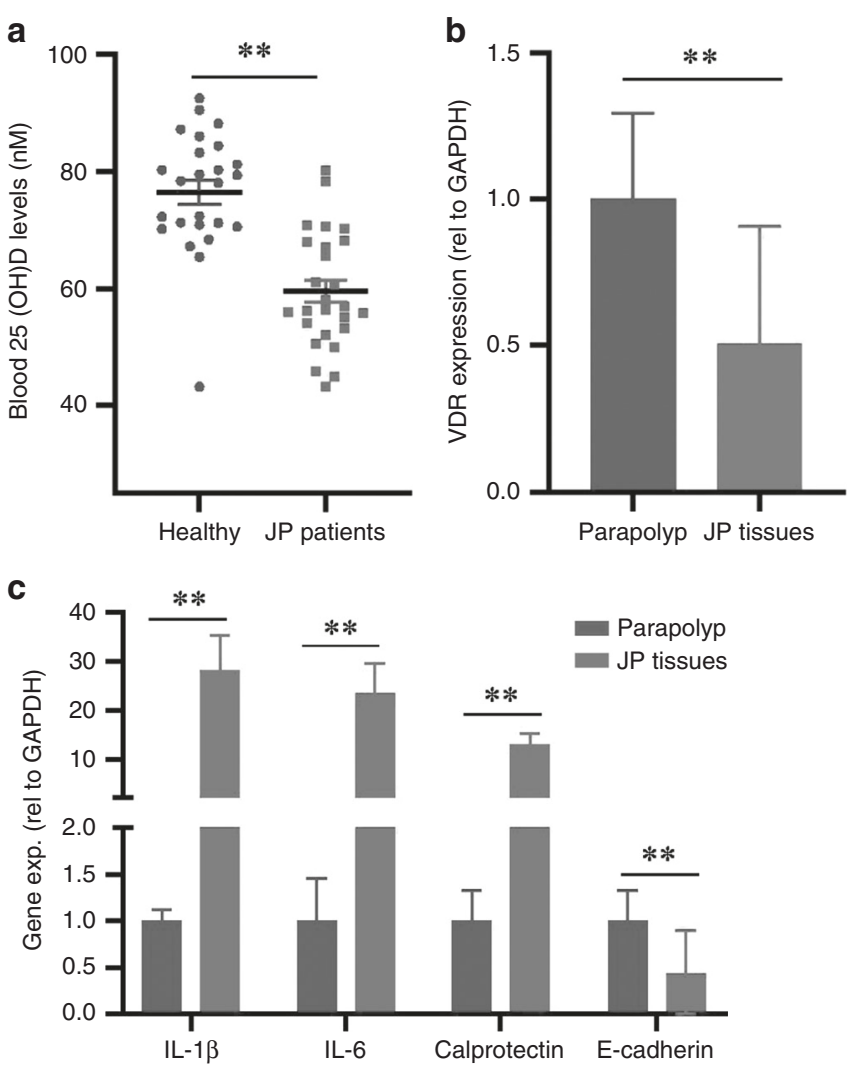

Fig. 1 Gene expression of IL-1 $\beta$, IL-6, calprotectin, E-cadherin, and VDR in juvenile polyp and parapolyp tissues. a $25(\mathrm{OH}) \mathrm{D}$ levels in the blood of the participants $(n=27)$ and in those of age- and sexpaired healthy individuals $(n=24)$ are plotted. The information includes 27 patients who had blood 25(OH)D levels tested prior to polypectomy. b, c Gene expression of IL-1 $\beta$, IL-6, and S109A subunit of calprotectin, E-cadherin, and VDR in the parapolyp specimens $(n=24)$ and the polyp tissues $(n=76)$ was detected by qPCR. GAPDH expression in individual samples was used for normalization. The levels of gene expression in the parapolyp tissues were arbitrarily assigned as 1 . A $P$ value of $\leq 0.05$ was considered statistically significant. ${ }^{*} P<0.01$.

that the level of $25(\mathrm{OH}) \mathrm{D}$ in the blood had no relationship with the VDR gene expression in the JP $(r=-0.1765,95 \% \mathrm{Cl},-0.8643$ to 0.7413). In addition, we also checked for IL-1 $\beta$, IL-6, calprotectin of inflammation, and E-cadherin of the tight junction. Compared to that of parapolyps, the JP tissues had decreased expression of VDR and E-cadherin. In contrast, the JP tissues had increased gene expression of IL-1 $\beta, I L-6$, and calprotectin, a marker for intestinal inflammation, ${ }^{32}$ indicating the existence of inflammation in the JP tissues (Fig. 1).

We then correlated HAdV prevalence to the expression of these genes in the polyp tissues (Table 3). By nonparametric Spearman's correlation analysis, we found that HAdV prevalence in the polyp tissues positively correlated to IL-1 $\beta, \mathrm{IL}-6$, and calprotectin expression. Samples with low expression of VDR or with E-cadherin had a high prevalence of HAdV infection. The samples with low VDR expression were more prevalent of HAdV DNA, while those with high VDR expression had a significantly lower detection rate of HAdV DNA ( $r=$ $1.261,95 \% \mathrm{Cl}, 1.017-1.563)$. For comparison, 30 of the adenoviral DNA-positive samples were from 61 low VDR expression samples compared to that of parapolyps, while the 15 samples with higher than control VDR expression only had three with adenoviral DNA, representing a positive rate of $49.2 \%$ and $20.0 \%$, respectively. VDR function has a critical role in antiviral and antimicrobial defence. We found that VDR expression has a negative correlation with
Table 3. Univariate analysis of HAdV prevalence to gene expression of VDR and inflammation.

\begin{tabular}{|c|c|c|}
\hline Viarable & HAdV status ${ }^{a}$ & OR $(95 \% \mathrm{CI})^{\mathrm{b}}$ \\
\hline \multirow[t]{2}{*}{ VDR $(H / L)$} & + & $0.321(0.112-0.935)$ \\
\hline & - & $1.724(1.211-2.413)$ \\
\hline \multirow[t]{2}{*}{ IL-1 $\beta(H / L)$} & + & 11.663 (3.892-34.964) \\
\hline & - & $0.167(0.075-0.361)$ \\
\hline \multirow[t]{2}{*}{ IL-6 (H/L) } & + & $4.594(2.286-9.252)$ \\
\hline & - & $0.281(0.151-0.5243)$ \\
\hline \multirow[t]{2}{*}{ Calprotectin $(\mathrm{H} / \mathrm{L})$} & + & $5.001(2.346-10.708)$ \\
\hline & - & $0.293(0.171-0.536)$ \\
\hline \multirow[t]{2}{*}{ E-cadherin $(H / L)$} & + & $0.285(0.141-0.576)$ \\
\hline & - & $2.432(1.521-3.906)$ \\
\hline
\end{tabular}

Gene expression was normalized to GAPDH expression using the average number of corresponding genes in parapolyps as 1 . The high and low $(\mathrm{H} / \mathrm{L})$ of expression in the juvenile polyps was designated as higher or lower than those in the parapolyps of corresponding genes.

a + means HAdV-positive samples, - means HAdV-negative samples.

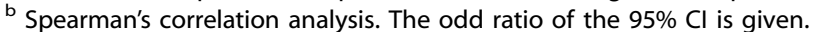

inflammation of IL-1 $\beta$, IL-6, and calprotectin expression (Fig. 2). The results thus suggest that low VDR expression is a risk factor for $\mathrm{Gl}$ HAdV infection and persistence, while low VDR expression positively correlated to the inflammation conditions of the samples.

In summary, we found for the first time that HAdV was common among JP samples. The rate of HAdV detection correlated positively with low VDR expression.

\section{DISCUSSION}

We screened the tissues by using PCR and found that the majority of JP tissues had HAdV. By detection of HAdV transcripts, we concluded that most of the patients were inactively infected, a conclusion that was supported by the detection of limited HAdV shedding in the stools. In this study, we detected subgroup $C$ viruses overwhelmingly from JPs. No HAd40 or Ad41 was detected from JP samples, although some studies reported that enteric HAdV (types 40 and 41) accounted for approximately half of the HAdV-positive cases in diarrheic children. ${ }^{18,33}$ The recent study seems to indicate that the HAdV subgroup $\mathrm{C}$ are common pathogens among children with enteric ailments.

HAdVs were first detected by Rowe and co-workers from removed adenoids and are known to cause a broad spectrum of human diseases. ${ }^{14}$ The viruses are classified into at least 51 serotypes within six species (A-F). It is now known that HAdV causes a wide spectrum of diseases, from uncomplicated acute respiratory to conjunctivitis and Gl infections in healthy individuals. Their role in Gl illnesses has been questioned until recently. Infants and children have considerable masses of lymphoid tissue. ${ }^{34,35}$ Stool virome in very early-onset inflammatory bowel disease shows a potential association of enteric viruses with inflammatory bowel disease. ${ }^{36}$ Serology and stool antigen testing show that patients with inflammatory bowel disease correlate with a viral infection. ${ }^{37}$ Intussusception is a type of intestinal invigoration. HAdV has been identified as a potential risk for developing intussusception in pediatric patients. In a study among Korean children with intussusception, subgroup $C$ nonenteric HAdVs were detected at a rate of $>95 \%$ and showed a significant epidemic correlation $(r=0.776 ; P=0.001)$ with intussusception. Those and our study show that subgroup C HAdV is a potential risk factor for intestinal diseases.

We found HAdV detection to be inversely correlated with low VDR expression. Low serum $25(\mathrm{OH}) \mathrm{D}$ concentration is associated with mucosal inflammation. ${ }^{38}$ Epidemiological studies support a link 

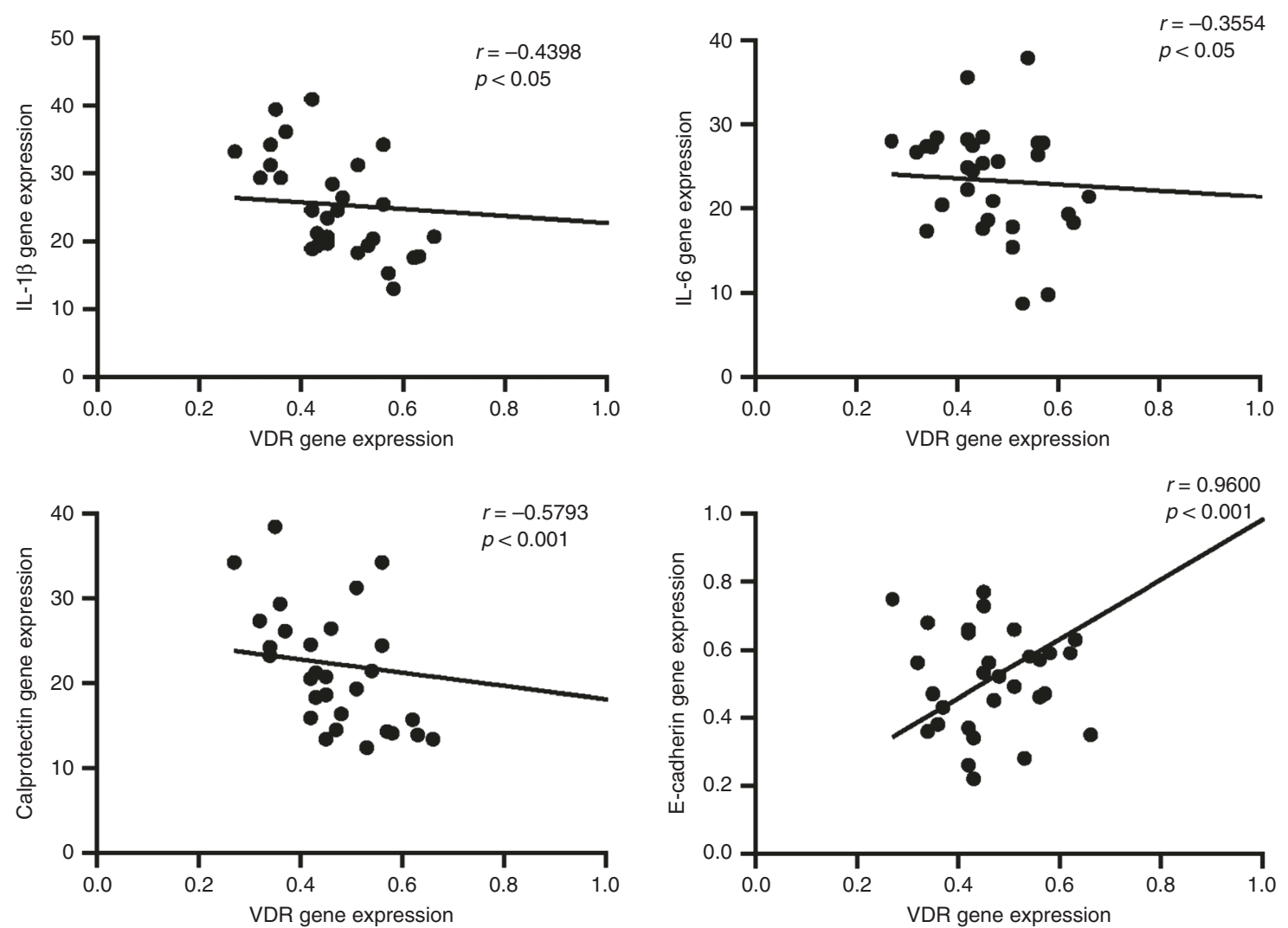

Fig. 2 Correlation analysis of VDR expression in association with IL-1 $\beta$, IL-6, calprotectin, and E-cadherin expression in HAdV-positive JPs. A Pearson's analysis was performed to correlate VDR expression to that of IL-1 $\beta$, IL-6, calprotectin, and E-cadherin.

between low 25(OH)D levels and a higher risk of viral infections. ${ }^{39,40}$ It was reported that vitamin D deficiency is prevalent among patients with HIV infection. ${ }^{41}$ SARS-CoV-2 positivity rates were higher among those with $25(\mathrm{OH}) \mathrm{D}$ deficiency. ${ }^{42}$ Although vitamin D supplementation did not reduce the overall wintertime upper respiratory tract infections among healthy children, ${ }^{43}$ vitamin D supplementation protected against acute respiratory tract infection among vitamin D-deficient patients. ${ }^{44}$ Vitamin $D$ decreases induction of NF-kB pathway-regulated chemokines and cytokines while maintaining the antiviral state against respiratory syncytial virus infection. ${ }^{45}$ Cell culture experiments support the thesis that vitamin $D$ has direct antiviral effects since vitamin D has the ability to upregulate antimicrobial peptides LL-37 and human beta-defensin 2 expressions. $^{44}$

Subgroup C HAdVs use Coxsackievirus and adenovirus receptor (CAR), a $46 \mathrm{kDa}$ transmembrane protein, for high-affinity binding to the host cells. CAR is a transmembrane component of the tight junction. Vitamin $D$ deficiency predisposes to adherent-invasive Escherichia coli-induced barrier dysfunction in the gut and attenuates the innate immune pathway of antimicrobial and anti-inflammatory response. ${ }^{46-48}$ In nonpolarized cells, CAR localized to homotypic intercellular contacts, mediated homotypic cell aggregation, and recruited the tight junction protein ZO-1 to sites of cell-cell contact. ${ }^{49}$ Whether reduced VDR expression contributes to increased incidence of virus infection that may exacerbate inflammation and intussusception bleeding remains unknown. Vitamin D deficiency promotes epithelial barrier dysfunction and intestinal inflammation. ${ }^{50,51}$ It has been reported that VDR deletion leads to the destruction of tight and adherent junctions in the lungs, ${ }^{52}$ while calcium and vitamin $D$ supplements have a positive effect on tight-junction proteins and mucin-12 expression in the normal rectal mucosa of colorectal adenoma patients. ${ }^{53}$ It is plausible to speculate that the dysfunction of which may lead to endotoxemia and inflammation, and contribute to increased incidence of virus infection and potentially reactivation.

Our study shows that nonproductive HAdV is common among JP tissues and uncovers a correlation between vitamin D deficiency and HAdV prevalence, highlighting the need for monitoring interventional studies on vitamin D status in young children with digestive system ailments in particular.

\section{REFERENCES}

1. Mestre, J. R. The changing pattern of juvenile polyps. Am. J. Gastroenterol. 81, 312-314 (1986).

2. Lelli, J. L. in Pediatric Surgery (ed. Coran, A. G.) 1177-1185 (Mosby, 2012).

3. Lee, Y. J. \& Park, J. H. The most common cause of lower gastrointestinal bleeding without other symptoms in children is colonic polyp: is total colonoscopy needed? Clin. Endosc. 52, 207-208 (2019).

4. Fox, V. L., Perros, S., Jiang, H. \& Goldsmith, J. D. Juvenile polyps: recurrence in patients with multiple and solitary polyps. Clin. Gastroenterol. Hepatol. 8, 795-799 (2010).

5. Cynamon, H. A., Milov, D. E. \& Andres, J. M. Diagnosis and management of colonic polyps in children. J. Pediatr. 114, 593-596 (1989).

6. Thakkar, K. et al. Prevalence of colorectal polyps in pediatric colonoscopy. Dig. Dis. Sci. 57, 1050-1055 (2012).

7. Lelli, J. L. in Pediatric Surgery 4th edn (eds Grosfeld, J. L., O'Neill, J. A., Coran, A. G., Fonkalsrud, E. W. \& Caldamone, A. A.) 1414-1426 (Mosby, 2006).

8. Kosulin, K. et al. Persistence and reactivation of human adenoviruses in the gastrointestinal tract. Clin. Microbiol Infect. 22, e381-e388 (2016).

9. Kosulin, K. Intestinal Hadv infection: tissue specificity, persistence, and implications for antiviral therapy. Viruses 11, 804 (2019).

10. Minney-Smith, C. A. et al. Intussusception is associated with the detection of adenovirus $C$, enterovirus $B$ and rotavirus in a rotavirus vaccinated population. $J$. Clin. Virol. 61, 579-584 (2014).

11. Bhisitkul, D. M., Todd, K. M. \& Listernick, R. Adenovirus infection and childhood intussusception. Am. J. Dis. Child 146, 1331-1333 (1992). 
12. Ukarapol, N. et al. Adenovirus infection: a potential risk for developing intussusception in pediatric patients. J. Med. Virol. 88, 1930-1935 (2016).

13. Bennett, S. \& Gunson, R. N. The development of a multiplex real-time RT-PCR for the detection of adenovirus, astrovirus, rotavirus and sapovirus from stool samples. J. Virol. Methods 242, 30-34 (2017).

14. Evans, A. S. Establishment of human adult tonsil cells in continuous culture and their virus susceptibilities. Proc. Soc. Exp. Biol. Med. 96, 752-757 (1957).

15. Garnett, C. T., Erdman, D., Xu, W. \& Gooding, L. R. Prevalence and quantitation of species $C$ adenovirus DNA in human mucosal lymphocytes. J. Virol. 76, 10608-10616 (2002).

16. Proenca-Modena, J. L. et al. Human adenovirus replication and persistence in hypertrophic adenoids and palatine tonsils in children. J. Med. Virol. 91, 1250-1262 (2019).

17. Wang, L. et al. Histone deacetylase inhibitors promote latent adenovirus reactivation from tonsillectomy specimens. J. Virol. 94, e00100-e00120 (2020).

18. Kumthip, K., Khamrin, P., Ushijima, H. \& Maneekarn, N. Enteric and non-enteric adenoviruses associated with acute gastroenteritis in pediatric patients in Thailand, 2011 to 2017. PLoS ONE 14, e0220263 (2019).

19. Lynch, J. P. 3rd \& Kajon, A. E. Adenovirus: epidemiology, global spread of novel serotypes, and advances in treatment and prevention. Semin. Respir. Crit. Care Med. 37, 586-602 (2016).

20. Roy, S. et al. Adenoviruses in lymphocytes of the human gastro-intestinal tract. PLOS ONE 6, e24859 (2011).

21. Okimoto, S., Hyodo, S., Yamamoto, M., Nakamura, K. \& Kobayashi, M. Association of viral isolates from stool samples with intussusception in children. Int. J. Infect. Dis. 15, e641-e645 (2011).

22. Nakamura, $\mathrm{N}$. et al. Molecular epidemiology of enteric viruses in patients with acute gastroenteritis in Aichi Prefecture, Japan, 2008/09-2013/14. J. Med. Virol. 88, 1180-1186 (2016)

23. Moyo, S. J. et al. Prevalence and molecular characterisation of human adenovirus in diarrhoeic children in Tanzania; a Case Control Study. BMC Infect. Dis. 14, 666 (2014).

24. Uhnoo, I., Svensson, L. \& Wadell, G. Enteric adenoviruses. Baillieres Clin. Gastroenterol. 4, 627-642 (1990).

25. Allard, A., Albinsson, B. \& Wadell, G. Rapid typing of human adenoviruses by a general PCR combined with restriction endonuclease analysis. J. Clin. Microbiol. 39, 498-505 (2001).

26. Bustin, S. A. et al. The MIQE guidelines: minimum information for publication of quantitative real-time PCR experiments. Clin. Chem. 55, 611-622 (2009).

27. Hossein-nezhad, A. \& Holick, M. F. Vitamin D for health: a global perspective. Mayo Clin. Proc. 88, 720-755 (2013).

28. Selvaraj, G., Kirkwood, C., Bines, J. \& Buttery, J. Molecular epidemiology of adenovirus isolates from patients diagnosed with intussusception in Melbourne, Australia. J. Clin. Microbiol. 44, 3371-3373 (2006).

29. Lappalainen, S. et al. Simultaneous presence of human herpesvirus 6 and adenovirus infections in intestinal intussusception of young children. Acta Paediatr. 101, 663-670 (2012)

30. Chen, C. et al. Bacteroides, butyric acid and T10,C12-Cla changes in colorectal adenomatous polyp patients. Gut Pathog. 13, 1 (2021).

31. Silva, L. G., Ferguson, B. S., Avila, A. S. \& Faciola, A. P. Sodium propionate and sodium butyrate effects on histone deacetylase (HDAC) activity, histone acetylation, and inflammatory gene expression in bovine mammary epithelial cells. J. Anim. Sci. 96, 5244-5252 (2018).

32. van Rheenen, P. F., Van de Vijver, E. \& Fidler, V. Faecal calprotectin for screening of patients with suspected inflammatory bowel disease: diagnostic meta-analysis. BMJ 341, c3369 (2010).

33. Banerjee, A., De, P., Manna, B. \& Chawla-Sarkar, M. Molecular characterization of enteric adenovirus genotypes 40 and 41 identified in children with acute gastroenteritis in Kolkata, India during 2013-2014. J. Med Virol. 89, 606-614 (2017).

34. Montgomery, E. A. \& Popek, E. J. Intussusception, adenovirus, and children: a brief reaffirmation. Hum. Pathol. 25, 169-174 (1994).

35. Forchielli, M. L. \& Walker, W. A. The role of gut-associated lymphoid tissues and mucosal defence. Br. J. Nutr. 93(Suppl. 1), S41-S48 (2005).

36. Liang, G. et al. Dynamics of the stool virome in very early-onset inflammatory bowel disease. J. Crohns Colitis 14, 1600-1610 (2020).

37. Gebhard, R. L. et al. Acute viral enteritis and exacerbations of inflammatory bowel disease. Gastroenterology 83, 1207-1209 (1982).

38. Meckel, K. et al. Serum 25-dydroxyvitamin D concentration is inversely associated with mucosal inflammation in patients with ulcerative colitis. Am. J. Clin. Nutr. 104, 113-120 (2016).

39. Griffin, G. et al. Vitamin D and Covid-19: evidence and recommendations for supplementation. R. Soc. Open Sci. 7, 201912 (2020).

40. Radujkovic, A. et al. Vitamin D deficiency and outcome of Covid-19 patients. Nutrients 12, 2757 (2020).
41. Jimenez-Sousa, M. A., Martinez, I., Medrano, L. M., Fernandez-Rodriguez, A. \& Resino, S. Vitamin D in human immunodeficiency virus infection: influence on immunity and disease. Front. Immunol. 9, 458 (2018).

42. Kaufman, H. W., Niles, J. K., Kroll, M. H., Bi, C. \& Holick, M. F. Sars-Cov-2 positivity rates associated with circulating 25-hydroxyvitamin D levels. PLOS ONE 15, e0239252 (2020).

43. Aglipay, M. et al. Effect of high-dose vs standard-dose wintertime vitamin D supplementation on viral upper respiratory tract infections in young healthy children. JAMA 318, 245-254 (2017).

44. Martineau, A. R. et al. Vitamin D supplementation to prevent acute respiratory tract infections: systematic review and meta-analysis of individual participant data. BMJ 356, i6583 (2017).

45. Hansdottir, S. et al. Vitamin D decreases respiratory syncytial virus induction of NF-kappaB-linked chemokines and cytokines in airway epithelium while maintaining the antiviral state. J. Immunol. 184, 965-974 (2010).

46. Assa, A. et al. Vitamin D deficiency predisposes to adherent-invasive Escherichia coli-induced barrier dysfunction and experimental colonic injury. Inflamm. Bowel Dis. 21, 297-306 (2015).

47. Makishima, M. et al. Vitamin D receptor as an intestinal bile acid sensor. Science 296, 1313-1316 (2002).

48. Liu, P. T. et al. Toll-like receptor triggering of a vitamin D-mediated human antimicrobial response. Science 311, 1770-1773 (2006).

49. Cohen, C. J. et al. The Coxsackievirus and adenovirus receptor is a transmembrane component of the tight junction. Proc. Natl Acad. Sci. USA 98, 15191-15196 (2001).

50. Assa, A. et al. Vitamin D deficiency promotes epithelial barrier dysfunction and intestinal inflammation. J. Infect. Dis. 210, 1296-1305 (2014).

51. Ardesia, M., Ferlazzo, G. \& Fries, W. Vitamin D and inflammatory bowel disease. Biomed. Res. Int. 2015, 470805 (2015).

52. Chen, H., Lu, R., Zhang, Y. G. \& Sun, J. Vitamin D receptor deletion leads to the destruction of tight and adherens junctions in lungs. Tissue Barriers 6, 1-13 (2018).

53. Mandle, H. B. et al. Effects of supplemental calcium and vitamin D on tightjunction proteins and mucin-12 expression in the normal rectal mucosa of colorectal adenoma patients. Mol. Carcinogen. 58, 1279-1290 (2019).

\section{AUTHOR CONTRIBUTIONS}

G.Y. and E.L. conceived the ideas, J.L., G.Y., and H.G. collected the samples, and L.W. and J.L. processed the samples and performed the studies. G.Y., J.L., S.H., and E.L. analyzed the data, and L.W. and E.L. prepared the first draft. All authors have read and approved the current version.

\section{FUNDING INFORMATION}

This work was financially supported by grants from Key Project of Research and Development of Ningxia Hui Autonomous Region (2017-BN04 to E.L.), from NSFC 81871636 and 81071859 to E.L.), from Nanjing Science and Technology (ZKX18040 to G.Y.), from Science, Technology and Innovation Commission of Shenzhen Municipality (JSGG 20200519160755008 to E.L.), from Fundamental Research Funds for the Central Universities (14380470 to S.H.), and from the Jiangsu Natural Science Foundation (BK20200316 to S.H.).

\section{COMPETING INTERESTS}

A human subject protocol was approved by the Ethics Committee of Nanjing Medical University and informed consent was obtained from parents or legal guardians in accordance with the Declaration of Helsinki. The authors declare no competing interests.

\section{INFORMED CONSENT}

Written informed parental consent or assent from all participants were obtained wherever necessary.

\section{ETHICS APPROVAL}

Approval of the institutional ethics committee was obtained (201412001-1).

\section{ADDITIONAL INFORMATION}

Correspondence and requests for materials should be addressed to G.Y. or E.L.

Reprints and permission information is available at http://www.nature.com/reprints

Publisher's note Springer Nature remains neutral with regard to jurisdictional claims in published maps and institutional affiliations. 